\title{
Schwinger Model Green functions with topological effects
}

\author{
Tomasz Radożycki* \\ Physics Department, Warsaw University, ul. Hoża 69, 00-681 Warsaw, Poland
}

\begin{abstract}
The fermion propagator and the 4-fermion Green function in the massless $\mathrm{QED}_{2}$ are explicitly found with topological effects taken into account. The corrections due to instanton sectors $k= \pm 1$, contributing to the propagator, are shown to be just the homogenous terms admitted by the Dyson-Schwinger equation for $S$. In the case of the 4 -fermion function also sectors $k= \pm 2$ are included into consideration. The quark condensates are then calculated and are shown to satisfy cluster property. The $\theta$-dependence exhibited by the Green functions corresponds to and may be removed by performing certain chiral gauge transformation.
\end{abstract}

11.10.-z;11.30.Rd;12.90.+b

Typeset using REVTEX

*Elecronic mail: torado@fuw.edu.pl 


\section{INTRODUCTION}

Many phenomena in present-day theory of elementary particles can be only described in the nonperturbative language. In the first place (though not exclusively) it refers to the theory of strong interactions — quantum chromodynamics — where the only existence of mesons and baryons requires allowing for such effects as quark and gluon confinement. However QCD, based on colour $S U(3)$ symmetry group, introduces a variety of coupled fields, which makes the perturbative calculation very complicated, and nonperturbative one extremely perplexed.

Fortunately we have at our disposal a couple of examples of model theories, that, thanks to their mathematical simplicity, allow for investigating certain nontrivial and nonperturbative aspects of more complicated phenomena. Among most fertile ones one should mention the so called Schwinger Model - the electrodynamics of massless fermions that, in this work, will be called 'quarks', in two space-time dimensions. This model was originally proposed [1] as an example of a field theory in which the existence of the local gauge symmetry does not entail the zero mass gauge boson - a fact which should not be pushed aside in electroweak interactions theory. It turned out soon, however, that it has many other nontrivial properties, which can be interesting from the point of view of both, strong and weak interactions. Above all one should mention here the confinement of fundamental fermions and the axial anomaly.

The similarity between quantum chromodynamics and the Schwinger Model appears also (and this will be particularly essential for this work) in the presence of topological effects: the existence of instanton sectors leading to the emerging of $\theta$-vacuum and nonzero 'quark' condensate.

It is a known fact [2] that if the theory contains massless fermions (at least one) the amplitude of the tunnelling transition between different topological vacua is diminished to zero. Mathematically it is expressed through zero value of the Euclidean Dirac operator determinant: $\operatorname{det}[i \not \partial-e \not A]$, because of the appearance of the zero eigenvalues and eigenvectors when $A$ bears the instanton index [3 7]. The functional integral over fermionic degrees of freedom, corresponding to the transition amplitude in question, is then zero and the tunnelling phenomenon disappears. On the other hand, however, the notion of the $\theta$-vacuum does not lose its sense since topological vacua do not exhibit the so called cluster property [8]. The reason is that, despite the appearance of the anomaly, a conserved chiral charge still can be defined, the topological vacua being its eigenstates with different eigenvalues. The matrix elements $<m|Q(x)| n>$ of certain local operator $Q(x)$ between such vacua $(\mid n>$ and $\mid m>)$ are then nonvanishing if the operator $Q$ changes the chirality (the Hamiltonian does not belong to that type of operators and hence the above mentioned lack of tunnelling). If one of the topological vacua were taken as the true vacuum, for such a type of operators $Q$ one would obtain automatically $\left\langle Q(x)>_{v a c}=0\right.$. A product of such operators $P(x, y)=Q_{1}(x) Q_{2}(y)$ can, however, have the nonzero vacuum expectation value, for instance if it is constructed in a chiral invariant way (e.g. if $Q_{1}$ changes the chirality by $k$ and $Q_{2}$ by $-k$ ). Now the requirement of clusterization in the limit $|x-y| \rightarrow \infty$ leads to contradiction [9]

$$
0 \neq<P>_{v a c}=<Q_{1}>_{v a c} \cdot<Q_{2}>_{v a c}=0 .
$$

If we then have to do with vacuum expectation values for chirality nonconserving operators, we have to include in the calculation different topological vacua. It is just that category that 
fermion, and fermion-boson Green functions belong to. Because of the $2: 1$ correspondence between the chiral charge and the topological index of a vacuum, the objects bilinear in fields $\Psi$ (propagators) require the inclusion of the instanton sectors $0, \pm 1$, and 4 -fermion functions - sectors $0, \pm 1, \pm 2$.

All these facts are, of course, well known and applied successfully to the calculations of the quark condensate $<\bar{\Psi}(x) \Psi(x)>_{\text {vac }}$ in the Schwinger Model, as well as to the verification of the revival of the cluster property for $<\bar{\Psi}(x) \Psi(x) \bar{\Psi}(y) \Psi(y)>_{v a c}$ [10 12].

The topological methods, however, have not been till now applied to the calculation of the full 2- and 4-point Green functions and not only condensates. This computation will constitute the main goal in the present work. The paper is organized in the following way. In Section II we concentrate on the quark propagator. From the considerations of the Dyson-Schwinger equations one knows [13,14 that, beside the famous Schwinger solution [1] satisfying $\left\{S, \gamma^{5}\right\}=0$ i.e. $S \sim \gamma^{\mu}(\ldots)_{\mu}$, they admit also other terms, let us call them $S^{\prime}$, for which $\left[S^{\prime}, \gamma^{5}\right]=0$. That means that $S^{\prime} \sim \gamma^{5}(\ldots)+\mathbb{1}(\ldots)$. The authors raised then the question concerning the interpretation of such terms. Extending slightly the analysis of [14] we show, in Section $1 \mathrm{~A}$, which the most general form of the quark propagator, resulting from the Dyson-Schwinger equations, is. Next, in Section II B using the methods of [10], applied there to find the value of condensate, we calculate the full propagator and show that the additional terms $S^{\prime}$ are just those emerging from nonzero instanton sectors. In section III we perform the similar calculation for the 4 -fermion function. The analysis is here more complicated since it now involves the $0, \pm 1, \pm 2$ instanton sectors and also because of the tensor structure of $G$, which bears now the four spinor indices. In the final section we verify the cluster property and have a look on the $\theta$ dependence of the full Green functions. As one knows, in massless theory the parameter $\theta$ may be removed by the convenient chiral gauge transformation. This conclusion will find its full confirmation in our expressions for the Green functions.

\section{INSTANTON CONTRIBUTIONS TO THE QUARK PROPAGATOR}

In this section we would like to concentrate on the fermion propagator. Being defined as the vacuum expectation value of the product of $\Psi$ and $\bar{\Psi}$ it should acquire additional terms, beside that found already by Schwinger, originating from instanton sectors \pm 1 .

We start with summarizing briefly the conventions used in this work. The twodimensional Lagrangian density of the Schwinger Model with the gauge fixing term has the following form

$$
\mathcal{L}(x)=\bar{\Psi}(x)\left(i \gamma^{\mu} \partial_{\mu}-e \gamma^{\mu} A_{\mu}(x)\right) \Psi(x)-\frac{1}{4} F^{\mu \nu}(x) F_{\mu \nu}(x)-\frac{\lambda}{2}\left(\partial_{\mu} A^{\mu}(x)\right)^{2},
$$

where for Dirac matrices $\gamma^{\mu}$ we assume the representation in which all gamma's are real

$$
\gamma^{0}=\left(\begin{array}{ll}
0 & 1 \\
1 & 0
\end{array}\right), \quad \gamma^{1}=\left(\begin{array}{rr}
0 & -1 \\
1 & 0
\end{array}\right), \quad \gamma^{5}=\gamma^{0} \gamma^{1}=\left(\begin{array}{rr}
1 & 0 \\
0 & -1
\end{array}\right),
$$

The metric tensor $g^{\mu \nu}$ and the totally antisymmetric symbol $\varepsilon^{\mu \nu}$ are defined as follows

$$
g^{00}=-g^{11}=1, \quad \varepsilon^{01}=-\varepsilon^{10}=1, \quad \varepsilon^{00}=\varepsilon^{11}=0 .
$$




\section{A. General considerations}

Having specified the Lagrangian we can derive, in the standard way, the Dyson-Schwinger equations [15, [16] for the propagators for the two basic fields in the theory. It is well known that the result for the gluon Green function is simply that it acquires a mass equal to $\sqrt{e^{2} / \pi}$. We recall that in two dimensions $e$ is a dimensionful constant. No other contributions from the nonzero instanton sectors come into play since $A^{\mu}$ does not change the chiral charge, similarly as interactions with photons do not change the electric charge (which is no more true in nonabelian theories). We, therefore, take the full, dressed gluon propagator as already known to be (in momentum space)

$$
D^{\mu \nu}(k)=\left(g^{\mu \nu}-\frac{k^{\mu} k^{\nu}}{k^{2}}\right) \frac{1}{e^{2} / \pi-k^{2}}-\frac{1}{\lambda} \frac{k^{\mu} k^{\nu}}{\left(k^{2}\right)^{2}},
$$

and concentrate only on the quark sector. The Dyson-Schwinger equation, as always, chains up the 2- and 3-point functions which recurrence (continued to infinity) usually efficiently precludes us from solving it

$$
\not p S(p)=1+i e^{2} \gamma^{\mu} \int \frac{d^{2} k}{(2 \pi)^{2}} S(p+k) \Gamma^{\nu}(p+k, p) S(p) D_{\mu \nu}(k) .
$$

The prominent and well known advantage of the Schwinger Model is that its Lagrangian is invariant under two types of gauge transformations: ordinary and chiral ones. This leads to two kinds of Ward identities [13, 17 19] which relate the projections of $\Gamma^{\mu}(p+k, p)$ on $k^{\mu}$ and on $\varepsilon^{\mu \nu} k_{\nu}$ with quark propagator. In two-dimensional world they are sufficient to reconstruct the full vertex $\Gamma^{\mu}$ and decouple the equation from the infinite hierarchy

$$
S(p+k) \Gamma^{\nu}(p+k, k) S(p)=\frac{k^{\nu}}{k^{2}}[S(p)-S(p+k)]-\frac{\varepsilon^{\nu \alpha} k_{\alpha}}{k^{2}}\left[\gamma^{5} S(p)+S(p+k) \gamma^{5}\right] .
$$

If we now adopt the Landau gauge $(\lambda \rightarrow \infty)$ we see that the longitudinal part of (5) does not contribute since $D^{\mu \nu}(k)$ becomes purely transverse and equation (4) may be given the following closed form

$$
\not p S(p)=1-i e^{2} \int \frac{d^{2} k}{(2 \pi)^{2}} \frac{1}{k^{2}\left(e^{2} / \pi-k^{2}-i \epsilon\right)} \not k \gamma^{5}\left[\gamma^{5} S(p)+S(p+k) \gamma^{5}\right],
$$

where the relation $\varepsilon^{\mu \alpha} \gamma_{\mu} k_{\alpha}=\not k \gamma^{5}$ has been used. In the co-ordinate space there is a known factorization and the equation is simplified to

$$
i \not \partial_{x} S(x)=\delta^{(2)}(x)-e^{2}\left[\partial_{x} \beta(x)\right] \gamma^{5} S(x) \gamma^{5}
$$

The function $\beta$ is here defined as follows

$$
\begin{aligned}
\beta(x) & =\int \frac{d^{2} p}{(2 \pi)^{2}}\left(1-e^{i p x}\right) \frac{1}{\left(p^{2}-e^{2} / \pi+i \epsilon\right)\left(p^{2}+i \epsilon\right)}= \\
& =\left\{\begin{array}{lll}
\frac{i}{2 e^{2}}\left[-\frac{i \pi}{2}+\gamma_{E}+\ln \sqrt{e^{2} x^{2} / 4 \pi}+\frac{i \pi}{2} H_{0}^{(1)}\left(\sqrt{e^{2} x^{2} / \pi}\right)\right] & x & \text { timelike } \\
\frac{i}{2 e^{2}}\left[\gamma_{E}+\ln \sqrt{-e^{2} x^{2} / 4 \pi}+K_{0}\left(\sqrt{-e^{2} x^{2} / \pi}\right)\right] & x & \text { spacelike },
\end{array}\right.
\end{aligned}
$$


and is, in fact, a function of $x^{2}$ only. Symbol $\gamma_{E}$ denotes the Euler constant and functions $H_{0}^{(1)}$ and $K_{0}$ are Hankel function of the first kind, and Basset function respectively [20. Assume now the most general matrical structure that is admitted in two dimensions, in co-ordinate space, for the fermion propagator $S$

$$
S(x)=\mathcal{S}_{0}(x) A\left(x^{2}\right)+B\left(x^{2}\right)+\gamma^{5} C\left(x^{2}\right)+\gamma^{5} \not \not D\left(x^{2}\right) .
$$

The term proportional to $\not x$ has been chosen, for convenience, as explicitly containing the free propagator $\mathcal{S}_{0}(x)=-\frac{1}{2 \pi} \frac{\not x}{x^{2}-i \varepsilon}$. It is, of course, only a question of redefining the coefficient function $A\left(x^{2}\right)$. In what follows we will omit epsilons specifying that we have to do with causal propagator. We now insert (9) into the co-ordinate space Dyson-Schwinger equation (7) and exploit the fact that for all unknown functions, as well as for $\beta$ function, we can write $\not_{x} F\left(x^{2}\right)=2 \not x d F / d x^{2}=2 \not x F^{\prime}\left(x^{2}\right)$. This allows us to deduce the set of four differential equations for four functions to be found. This set arises if one takes the trace of (17) with successive insertion of $\mathbb{1}, \gamma^{5}, \not x$ and $\gamma^{5} \not x$ on both sides. These are simple first order equations

$$
A^{\prime}=-i e^{2} \beta^{\prime} A, \quad B^{\prime}=i e^{2} \beta^{\prime} B, \quad C^{\prime}=i e^{2} \beta^{\prime} C, \quad D^{\prime}=-\left[\frac{1}{x^{2}}+i e^{2} \beta^{\prime}\right] D
$$

with the initial condition $A(0)=1$ originating from the cancellation of the Dirac delta functions in (7). This set of equations may immediately be solved and we obtain the most general form of the propagator that is accepted by the Dyson-Schwinger equation

$$
S(x)=\mathcal{S}_{0}(x) e^{-i e^{2} \beta(x)}+B_{0} e^{i e^{2} \beta(x)}+C_{0} \gamma^{5} e^{i e^{2} \beta(x)}+D_{0} \frac{\gamma^{5} \not x}{x^{2}} e^{-i e^{2} \beta(x)},
$$

where constants $B_{0}, C_{0}, D_{0}$ remain unknown.

In what was stated above we did not move to far from what had been done in [14], except that we found two additional terms $(C$ and $D)$ in the most general structure of $S$. In what follows, however, we will show that the terms with unknown constants in (11), except for the last one, arise as a result of instanton effects. The first term is just the well known Schwinger solution.

\section{B. Explicit calculation of the quark propagator}

The instanton contributions to the quark condensate were calculated in a systematic way in [10]. Below we extend this calculation and find the expression for the full propagator. We begin with substituting into the generating functional, defined as usually as

$$
Z[\eta, \bar{\eta}, J]=\int \mathcal{D} \Psi \mathcal{D} \bar{\Psi} \mathcal{D} A e^{i \int d^{2} x\left[\mathcal{L}+\bar{\eta} \Psi+\bar{\Psi} \eta+J^{\mu} A_{\mu}\right]}
$$

the following form of the gauge potential

$$
A^{\mu}(x)=A^{(0) \mu}(x)+\varepsilon^{\mu \nu} \partial_{\nu} b(x)
$$

where $A^{(0) \mu}$ is certain new potential satisfying some special conditions at space-time infinity (this point will be discussed afterwards) and $b$ is the external scalar function to be chosen 
later for our convenience. Since (13) constitutes a simple shift we can now easily pass from the functional integral over $A$ to that over $A^{(0)}$. It is known that the coupling term $e \bar{\Psi} \not A^{(0)} \Psi$ may be gauged away if we introduce new fermion fields defined by the relations

$$
\begin{aligned}
& \Psi(x)=e^{-i e \not \not_{x} \int d^{2} z \triangle(x-z) \gamma^{\mu} A_{\mu}^{(0)}(z)} \Psi^{\prime}(x) \stackrel{\text { def }}{=} e^{-i \phi\left(x, A^{(0)}\right)} \Psi^{\prime}(x), \\
& \Psi(x)=\bar{\Psi}^{\prime}(x) e^{i e \gamma^{\mu} \not_{x} \int d^{2} z \Delta(x-z) A_{\mu}^{(0)}(z) \stackrel{\text { def }}{=}} \bar{\Psi}^{\prime}(x) e^{i \tilde{\phi}\left(x, A^{(0)}\right)},
\end{aligned}
$$

with $\triangle(x-z)$ satisfying: $\square_{x} \triangle(x-z)=\delta^{(2)}(x-z)$. The above gauge transformation is an element of $U(1) \otimes U_{A}(1)$ group. While the Lagrangian (11) is invariant (apart from the gauge fixing term) under that kind of transformations, the fermion path integral measure in (12) is not 21 23]. This anomalous behaviour generates a mass term for the gauge boson. After this transformation is performed in (12), the $A^{(0)}$ dependence reappears in the source terms through the functions $\phi\left(x, A^{(0)}\right)$ and $\tilde{\phi}\left(x, A^{(0)}\right)$ defined by equations (14, 15). The substitution $A^{(0)} \rightarrow \frac{\delta}{i \delta J}$ allows us to perform the remaining gaussian integral over $A^{(0)}$ [24] and we obtain the following expression for $Z$

$$
\begin{aligned}
& Z[\eta, \bar{\eta}, J]=N_{b} \exp \left\{i \int d^{2} x\left[\frac{1}{2} b \square^{2} b+\varepsilon^{\mu \nu} J_{\mu} \partial_{\nu} b\right]\right\} \cdot \int \mathcal{D} \Psi \mathcal{D} \bar{\Psi} \times \\
& \exp \left\{i \int d^{2} x\left[\bar{\Psi}\left(i \not \partial-e \varepsilon^{\mu \nu} \gamma_{\mu} \partial_{\nu} b\right) \Psi+\bar{\eta} e^{-i \phi(x, \delta / i \delta J)} \Psi+\bar{\Psi} e^{i \tilde{\phi}(x, \delta / i \delta J)} \eta\right]\right\} \times \\
& \exp \left\{-\frac{i}{2} \int d^{2} x d^{2} y\left[J^{\mu}+\left(\square+e^{2} / \pi\right) \varepsilon^{\mu \alpha} \partial_{\alpha} b\right] \triangle_{\mu \nu}\left(x-y, e^{2} / \pi\right)\left[J^{\nu}+\left(\square+e^{2} / \pi\right) \varepsilon^{\nu \beta} \partial_{\beta} b\right]\right\},
\end{aligned}
$$

where $N_{b}$ is certain constant ( $b$ stands for boson). The massive propagator $\triangle^{\mu \nu}\left(x-y, e^{2} / \pi\right)$ satisfies the equation

$$
\left[\left(\square+\frac{e^{2}}{\pi}\right)\left(g_{\mu \nu}-\frac{\partial_{\mu} \partial_{\nu}}{\square}\right)+\lambda \partial_{\mu} \partial_{\nu}\right] \triangle^{\nu \alpha}\left(x-y, e^{2} / \pi\right)=\delta^{(2)}(x-y) g_{\mu}^{\alpha} .
$$

The main point is now the evaluation of the fermion path integral in (16). Let us denote by $X$ the following expression

$$
X=\int \mathcal{D} \Psi \mathcal{D} \bar{\Psi} \exp \left\{i \int d^{2} x\left[\bar{\Psi}\left(i \not \partial-e \varepsilon^{\mu \nu} \gamma_{\mu} \partial_{\nu} b\right) \Psi+\bar{\eta}^{\prime} \Psi+\bar{\Psi} \eta^{\prime}\right]\right\}
$$

where primes are used to avoid writing explicitly the factors $e^{-i \phi}$ multiplying the external sources. This expression is naturally proportional to the determinant of the Dirac operator. From (18) it is evident, however, that it strongly depends on the choice of hitherto undefined function $b$. It turns out that for certain choices of $b$ it may even vanish so one could proceed here with caution. First of all we temporarily go over to the Euclidean space since the properties of the Dirac operator are there mathematically more rigorous. The transition to this space is defined by the following substitutions: $x^{0} \rightarrow-i x_{2}, A^{0} \rightarrow i A_{2}, \partial^{0} \rightarrow i \partial_{2}$. The Euclidean metric tensor $g_{\mu \nu}$ is defined as $\delta_{\mu \nu}$ and for $\varepsilon_{\mu \nu}$ we have $\varepsilon_{12}=-\varepsilon_{21}=-i$. In what follows the same symbols as before will be used for denoting the Euclidean counterparts of earlier defined quantities. We hope that it will not cause any confusion and the passing back to the Minkowski space will explicitly be stated. Before we calculate the quantity (18) we have to make some remarks on the instanton sectors. 
Consider the most general form of the field $A$ in the 2-dimensional world

$$
A_{\mu}(x)=\partial_{\mu} a(x)-\varepsilon_{\mu \nu} \partial_{\nu} b(x),
$$

The so called Pontryagin index for the gluon field may be defined as

$$
\nu=\frac{i e}{4 \pi} \int d^{2} x \varepsilon_{\alpha \beta} F_{\alpha \beta}=-\frac{i e}{2 \pi} \int d^{2} x \square_{x} b(x) .
$$

Due to the Euclidean nature of the space-time the d'Alambert operator $\square_{x}$ is here, naturally, the same as the Laplace operator. Assume now that we take the function $b(x)$ in certain specific form

$$
b^{(k)}(x)=\frac{i}{2 e} k \ln \left(\frac{x^{2}+\lambda^{2}}{\lambda^{2}}\right),
$$

where $k$ is an integer number. After evaluation of (20) we immediately obtain $\nu=k$ i.e. the field $A_{\alpha}^{(k)}$ defined as $\varepsilon_{\alpha \beta} \partial_{\beta} b^{(k)}$ bears the index $\nu=k$. Since the Pontryagin index (20) is linear in gluonic field the total index of the sum

$$
A_{\alpha}^{(0)}+\varepsilon_{\alpha \beta} \partial_{\beta} b^{(k)},
$$

is equal to $k$, and thanks to the complete freedom while choosing $A^{(0)}$ (which is restricted to the sector 0 ) constitutes the most general form of the field in the $k$ instanton sector: $A_{\alpha}^{(k)}$. $A_{\alpha}^{(k)}$ represents a path (in the sense of Feynman path integal) connecting two distinct and topologically inequivalent vacua for $t= \pm \infty$. The true vacuum of the theory, the so called $\theta$-vacuum, is now the superposition of these topological vacua

$$
\left|\theta>=\sum_{n=-\infty}^{\infty} e^{i n \theta}\right| n>,
$$

and the generating functional calculated in this new vacuum has the form

$$
Z[\eta, \bar{\eta}, J]=\sum_{k=-\infty}^{\infty} e^{i k \theta} Z^{(k)}[\eta, \bar{\eta}, J]
$$

where the summation runs over instanton indices rather than over topological vacua ones. In each sector the appropriate $Z^{(k)}$ is calculated with the restriction on the values of the vector potential to those defined by (22). Now we are ready for considering the contributions from separate terms of the sum in (24) - the different instanton sectors. In compliance with what was said in the Introduction about chirality conserving and nonconserving operators and their matrix elements taken between different topological vacua, we have $Z[0,0,0]=$ $Z^{(0)}[0,0,0] \stackrel{\text { def }}{=} N_{f} \cdot N_{b}$ and for the propagator we can immediately write

$$
S(x-y)=\sum_{k=-\infty}^{\infty} S^{(k)}(x-y)=-\left.i \frac{1}{Z^{(0)}[0,0,0]} \sum_{k=-\infty}^{\infty} e^{i k \theta} \frac{\delta^{2} Z^{(k)}[\eta, \bar{\eta}, J]}{\delta \bar{\eta}(x) \delta \eta(y)}\right|_{J, \eta, \bar{\eta}=0} .
$$

Similar formulae are valid also for other Green functions and will be exploited in the following section while dealing with 4-point functions. The sum in (25) formally extends to $+\infty$ but 
practically depends on the properties of the operator the vacuum expectation value of which is being considered. In the case of a propagator this operator is simply a product $\Psi \bar{\Psi}$ and the whole sum reduces to three terms corresponding to $k=0, k=+1$ and $k=-1$ which means that chiral charge of the contributing vacua can, at most, differ by 2 . It is not difficult to observe that the case $k=0$ corresponds to the well known Schwinger solution [1], since it simply requires putting $b^{(k)}=0$, and is represented by the first term of (11). The nontrivial topological effects come from two other sectors. We concentrate below on the sector $k=+1$, the calculation for $k=-1$ being analogous. The applied method is here that of 10 and therefore we do not plunge into details and point out only the main steps.

For $k=1$ we have to substitute $b=b^{(1)}$ into (18) (or rather into its Euclidean version) and perform the $\Psi$ and $\bar{\Psi}$ functional integral. By the Atiyah-Singer index theorem [3 [7] the massless Dirac operator in the external field bearing the nonzero Pontryagin number possesses zero modes. For $k= \pm 1$ there is only such mode and, for gamma matrices conventions defined by (2), it has the form (for the discussion and construction of the zero modes in the Schwinger Model see [10,25,26])

$$
\begin{aligned}
& \chi_{0}(x)=\frac{1}{\sqrt{2 \pi}}\left(\frac{1}{\lambda^{2}+x^{2}}\right)^{1 / 2}\left(\begin{array}{l}
0 \\
1
\end{array}\right) \quad \text { for } k=+1, \\
& \chi_{0}(x)=\frac{1}{\sqrt{2 \pi}}\left(\frac{1}{\lambda^{2}+x^{2}}\right)^{1 / 2}\left(\begin{array}{l}
1 \\
0
\end{array}\right) \text { for } k=-1,
\end{aligned}
$$

In the 2-instanton sector, which will be dealt with in Section III], two zero modes appear

$$
\begin{aligned}
& \chi_{0}(x)=\frac{1}{\sqrt{2 \pi}} \frac{\lambda^{3 / 2}}{\lambda^{2}+x^{2}}\left(\begin{array}{l}
0 \\
1
\end{array}\right), \quad \chi_{1}(x)=\frac{1}{\sqrt{2 \pi}}\left(x_{1}-i x_{2}\right) \frac{\lambda^{1 / 2}}{\lambda^{2}+x^{2}}\left(\begin{array}{l}
0 \\
1
\end{array}\right) \quad \text { for } k=+2, \\
& \chi_{0}(x)=\frac{1}{\sqrt{2 \pi}} \frac{\lambda^{3 / 2}}{\lambda^{2}+x^{2}}\left(\begin{array}{l}
1 \\
0
\end{array}\right), \quad \chi_{1}(x)=\frac{1}{\sqrt{2 \pi}}\left(x_{1}+i x_{2}\right) \frac{\lambda^{1 / 2}}{\lambda^{2}+x^{2}}\left(\begin{array}{l}
1 \\
0
\end{array}\right) \quad \text { for } k=-2,
\end{aligned}
$$

The whole set of eigenmodes will be denoted by $\chi_{n}$. We have

$$
\Psi=\sum_{n} a_{n} \chi_{n}, \quad \bar{\Psi}=\sum_{n} b_{n} \chi_{n}^{+},
$$

where $a_{n}$ and $b_{n}$ are the Grassmann coefficients. We now pass from the integration over $\Psi$ and $\bar{\Psi}$ to that over $a_{n}$ and $b_{n}$. The presence of a zero mode means that neither $a_{0}$ nor $b_{0}$ appear in the bilinear term in the exponent of (18) and only source terms in the integration over these two parameters are involved. The rules for the Grassmann integration demand, however, that from the whole exponent only the linear part contribute. The integration over nonzero modes may be done in usual way, the details of which can be found in [10]. In that way we arrive at the following expression for the quantity $X$

$$
\begin{aligned}
X= & N_{f} \lambda \int d^{2} x d^{2} y \bar{\eta}^{\prime}(x) \chi_{0}(x) \chi_{0}^{+}(y) \eta^{\prime}(y) \exp \left[-\int d^{2} x d^{2} y \bar{\eta}^{\prime}(x) e^{-i \phi\left(x,-\varepsilon_{\mu \nu} \partial_{\nu} b^{(1)}\right)}\right. \\
& \left.S_{0}(x-y) e^{i \tilde{\phi}\left(y,-\varepsilon_{\mu \nu} \partial_{\nu} b^{(1)}\right)} \eta^{\prime}(y)\right] \cdot \exp \left[-\int d^{2} x \frac{e^{2}}{2 \pi} b^{(1)} \square b^{(1)}\right],
\end{aligned}
$$

$S_{0}$ being the Euclidean free fermion propagator: $i \not \partial_{x} S_{0}(x-y)=-\delta^{(2)}(x-y)$. The additional factor $\lambda$ arises from the change of variables (30) since the dimension of field $\Psi$ in the Feynman path integral measure is $1 / 2$ and that of $a_{0}$ and $b_{0}-0$. 
Now, when the integration over fermionic degrees of freedom has been performed, and the properties of the Euclidean Dirac operator have been exploited, it is convenient to come back to Minkowski space, where the final formula for the propagator, analogous to (11), is to be obtained. It should, however, be emphasized that whenever required by the mathematical rigour, the corresponding expressions in Euclidean space are presumably considered.

The differentiation over external currents $\eta$ and $\bar{\eta}$, as required by (25), leads to a very simple expression if one keeps in mind that finally we have to set all the external sources to zero

$$
\left.\frac{\delta^{2} X}{\delta \bar{\eta}(x) \delta \eta(y)}\right|_{\eta, \bar{\eta}=0}=-N_{f} \lambda e^{-i \phi(x, \delta / i \delta J)} \chi_{0}(x) \chi_{0}^{+}(y) e^{i \tilde{\phi}(y, \delta / i \delta J)} e^{i \int d^{2} x \frac{e^{2}}{2 \pi} b^{(1)} \square b^{(1)}}
$$

From the definition (26) of the zero mode it is evident that the product $\chi_{0} \chi_{0}^{+}$has the matrical form $\frac{1}{2}\left(\mathbb{1}-\gamma^{5}\right)$. The functional $\phi$ has similar structure (a linear combination of $\mathbb{1}$ and $\gamma^{5}$ ) and therefore both quantities commute with each other which leads to the considering of the operator

$$
\exp [-i \phi(x, \delta / i \delta J)+i \tilde{\phi}(y, \delta / i \delta J)],
$$

where functional differentiations act on the $J$ dependent argument of the last exponent function in (16). Let us now introduce the (nonconserved) current $\mathcal{K}^{\mu}$ by the relation

$$
\phi(x, \mathcal{A})-\tilde{\phi}(y, \mathcal{A})=-\int d^{2} z \mathcal{A}^{\mu}(z) \mathcal{K}_{\mu}(z ; x, y),
$$

and satisfying

$$
\partial_{z}^{\mu} \mathcal{K}_{\mu}(z ; x, y)=e\left[\delta^{(2)}(x-z)-\delta^{(2)}(y-z)\right],
$$

which is evident by virtue of the definitions (14.15) of $\phi$ and $\tilde{\phi}$. In this notation the operator (33) is just: $\exp \left[\int d^{2} z \mathcal{K}^{\mu}(z ; x, y) \delta / \delta J^{\mu}(z)\right]$ and simply shifts the argument of the $J$ dependent functional

$$
\exp \left[\int d^{2} z \mathcal{K}^{\mu}(z ; \cdot, \cdot) \frac{\delta}{\delta J^{\mu}(z)}\right] F[J]=F[J+\mathcal{K}] .
$$

Having found expression (32) for the second derivative of $X$, and collecting (16) together with (25) we are in a position to write down the formula for the one-instanton contribution to the quark propagator

$$
\begin{aligned}
& S^{(1)}(x-y)=i \lambda e^{i \theta} \chi_{0}(x) \chi_{0}^{+}(y) \cdot \exp \left\{\frac{i}{2} \int d^{2} u\left[b^{(1)}(u) \square\left(\square+e^{2} / \pi\right) b^{(1)}(u)\right]\right. \\
& -\frac{i}{2} \int d^{2} u d^{2} w\left[\mathcal{K}^{\mu}(u ; x, y)+\varepsilon^{\mu \alpha}\left(\square+e^{2} / \pi\right) \partial_{\alpha} b^{(1)}(u)\right] \triangle_{\mu \nu}(u-w)\left[\mathcal{K}^{\nu}(w ; x, y)+\right. \\
& \left.\left.\varepsilon^{\nu \beta}\left(\square+e^{2} / \pi\right) \partial_{\beta} b^{(1)}(w)\right]\right\} .
\end{aligned}
$$

In the exponent of (37) all functions are perfectly known, so it is not difficult, although lengthy, to evaluate all expressions. We skip this calculation here and only note that all terms may be divided onto three groups: those not containing current $\mathcal{K}^{\mu}$, linear, and finally quadratic in $\mathcal{K}^{\mu}$. 
1. It is not difficult to check that both terms which do not depend on $\mathcal{K}$ cancel each other.

2. The terms linear in $\mathcal{K}$ are strongly simplified if one notices that thanks to the presence of $\varepsilon^{\mu \nu}$ from the whole expression for $\triangle_{\mu \nu}$ only that proportional to metric tensor contributes. If one now exploit the known identity for gamma matrices in two dimensions $\gamma^{\beta} \gamma^{5}=\varepsilon^{\mu \beta} \gamma_{\mu}$ and observe that finally $\gamma^{5}$ may be replaced with -1 , because of the structure of the matrical coefficient $\left(\chi_{0} \chi_{0}^{+}\right)$in (37), we see that the total effect of these terms is just to cancel $\chi_{0} \chi_{0}^{+}$(up to $\frac{1}{2 \pi}$ ) together with the coefficient $\lambda$. This was, naturally, expected since the conclusive formula should not contain any trace of the particular choice of $b^{(1)}$.

3. The calculation of the term quadratic in $\mathcal{K}$ is also elementary and the result turns out to be

$$
\gamma_{E}+\frac{1}{2} \ln \frac{e}{2 \sqrt{\pi}}+i e^{2} \beta(x-y)
$$

This allows us to complete the formula (37) for $S^{(1)}$. The twin calculation in the $k=-1$ instanton sector gives the analogous outcome with the reversed signs of parameter $\theta$ and matrix $\gamma^{5}$. Finally, the quark propagator with contributions from all sectors is

$$
S(x)=\mathcal{S}_{0}(x) e^{-i e^{2} \beta(x)}+\frac{i e}{4 \pi^{3 / 2}}\left(\cos \theta-i \gamma^{5} \sin \theta\right) e^{\gamma_{E}+i e^{2} \beta(x)} .
$$

This proves that the second and the third term in (11), admitted by the Dyson-Schwinger equation, do actually materialize as a result of instanton effects. The last term does not reappear in (39), and in fact may not do, by virtue of simple dimensional consideration. From (11) it is obvious that the constant $D_{0}$ has to be dimensionless and simultaneously it must tend to zero when $e^{2} \rightarrow 0$ (the free case). Naturally no such constant exists in a world in which the only dimensionful constant is just $e^{2}$. From similar dimensional analysis it is obvious that constants $B_{0}$ and $C_{0}$ in (11) must be linear in $e$ which is in fact the case in (39). One should note in this point that instanton effects usully manifest themselves in a nonperturbative way, for instance through the appearance of negative powers of the coupling constant. As an example we can quote the one-instanton tunelling amplitude in four-dimensional YM: $\langle 0| 1>\sim \exp \left(-8 \pi^{2} / g^{2}\right)$ [27]. In the present case, however, we cannot construct $e^{2}$-dependent dimensionless constants, and the intuition concerning nonperturbative contributions should be confronted with dimensional considerations. In the last section we come back to the formula (39) and analyze it from the point of view of the quark condensate.

It should be also observed that other functions with two quark 'legs', as for instance the vertex function, are still completely expressible through the propagator $S$ as discussed in [19] and the relation

$$
\left[S \Gamma_{\mu} S\right]^{(k)}(x, y ; z)=i S^{(k)}(x-y) \gamma^{\mu} \not \partial_{z} \triangle(y-z)-i \not \partial_{z} \gamma^{\mu} \triangle(x-z) S^{(k)}(x-y),
$$

as well as its counterparts for higher functions, hold separately in each instanton sector. 


\section{INSTANTON CONTRIBUTIONS TO THE 4-FERMION FUNCTION}

In compliance with our former discussion, the expectation value of the product $\Psi \Psi \overline{\Psi \Psi}$ acquires contributions from up to $k= \pm 2$ instanton sectors. As in the case of the propagator, the 4 -fermion Green function in a certain specific sector is defined by the functional derivative of the generating functional

$$
G_{a b, c d}^{(k)}\left(x_{1}, x_{2} ; x_{3}, x_{4}\right)=\left.e^{i k \theta} \frac{1}{Z[0,0,0]} \frac{\delta^{4} Z^{(k)}[\eta, \bar{\eta}, J]}{\delta \bar{\eta}_{a}\left(x_{1}\right) \delta \bar{\eta}_{b}\left(x_{2}\right) \delta \eta_{c}\left(x_{3}\right) \delta \eta_{d}\left(x_{4}\right)}\right|_{\eta, \bar{\eta}, J=0} .
$$

For the sector $k=0$ the appropriate expression for the Green function was found in our previous work [19]. We, therefore, begin with considering the case $k=1$ recalling here only the final result for $G^{(0)}$

$$
\begin{aligned}
& G_{a b ; c d}^{(0)}\left(x_{1}, x_{2} ; x_{3}, x_{4}\right)=\frac{1}{2}\left[\mathcal{S}_{0 a c}\left(x_{1}-x_{3}\right) \mathcal{S}_{0 b d}\left(x_{2}-x_{4}\right)+\left(\mathcal{S}_{0}\left(x_{1}-x_{3}\right) \gamma^{5}\right)_{a c}\left(\mathcal{S}_{0}\left(x_{2}-x_{4}\right) \gamma^{5}\right)_{b d}\right] \\
& \exp \left[i e^{2}\left(\beta\left(x_{1}-x_{2}\right)-\beta\left(x_{1}-x_{3}\right)-\beta\left(x_{2}-x_{4}\right)-\beta\left(x_{1}-x_{4}\right)-\beta\left(x_{2}-x_{3}\right)+\beta\left(x_{3}-x_{4}\right)\right)\right] \\
& +\frac{1}{2}\left[\mathcal{S}_{0 a c}\left(x_{1}-x_{3}\right) \mathcal{S}_{0 b d}\left(x_{2}-x_{4}\right)-\left(\mathcal{S}_{0}\left(x_{1}-x_{3}\right) \gamma^{5}\right)_{a c}\left(\mathcal{S}_{0}\left(x_{2}-x_{4}\right) \gamma^{5}\right)_{b d}\right] \\
& \exp \left[-i e^{2}\left(\beta\left(x_{1}-x_{2}\right)-\beta\left(x_{1}-x_{3}\right)-\beta\left(x_{2}-x_{4}\right)-\beta\left(x_{1}-x_{4}\right)-\beta\left(x_{2}-x_{3}\right)+\beta\left(x_{3}-x_{4}\right)\right)\right] \\
& -\left(c, x_{3} \longleftrightarrow d, x_{4}\right) .
\end{aligned}
$$

The evaluation of $Z^{(1)}$ has already been done in the previous section so we are able to immediately write down the following equation for $G^{(1)}$

$$
\begin{aligned}
& G_{a b, c d}^{(1)}\left(x_{1}, x_{2} ; x_{3}, x_{4}\right)=i \lambda e^{i \theta}\left\{\left[( e ^ { - i \phi ( x _ { 1 } , \delta / i \delta J ) } \chi _ { 0 } ( x _ { 1 } ) \chi _ { 0 } ^ { + } ( x _ { 3 } ) e ^ { i \tilde { \phi } ( x _ { 3 } , \delta / i \delta J ) } ) _ { a c } \left(e^{-i \phi\left(x_{2}, \delta / i \delta J\right)}\right.\right.\right. \\
& \left.\left.\left.e^{-i \phi\left(x_{2}, \varepsilon^{\mu \nu} \partial_{\nu} b^{(1)}\right)} \mathcal{S}_{0}\left(x_{2}-x_{4}\right) e^{i \tilde{\phi}\left(x_{4}, \varepsilon^{\mu \nu} \partial_{\nu} b^{(1)}\right)} e^{i \tilde{\phi}\left(x_{4}, \delta / i \delta J\right)}\right)_{b d}-\left(c, x_{3} \leftrightarrow d, x_{4}\right)\right]-\left(a, x_{1} \leftrightarrow b, x_{2}\right)\right\} \\
& \exp \left[\frac{i}{2} \int d^{2} x b^{(1)} \square\left(\square+e^{2} / \pi\right) b^{(1)}-\frac{i}{2} \int d^{2} x d^{2} y\left(J^{\mu}(x)+\varepsilon^{\mu \alpha}\left(\square+e^{2} / \pi\right) \partial_{\alpha} b^{(1)}(x)\right)\right. \\
& \left.\triangle_{\mu \nu}(x-y)\left(J^{\nu}(y)+\varepsilon^{\nu \beta}\left(\square+e^{2} / \pi\right) \partial_{\beta} b^{(1)}(y)\right)\right]
\end{aligned}
$$

taken at $J=0$. We now recall the definition (34) of the current $\mathcal{K}$ from the preceding section and $\mathcal{J}$ introduced in 19$]$ by the relation

$$
\tilde{\phi}(x, \mathcal{A})-\tilde{\phi}(y, \mathcal{A})=-\int d^{2} z \mathcal{A}^{\mu}(z) \mathcal{J}_{\mu}(z ; x, y) .
$$

The both currents satisfy the equation

$$
\mathcal{K}^{\mu}(z ; x, y)=\mathcal{J}^{\mu}(z ; x, y)-2 e \gamma^{5} \varepsilon^{\mu \nu} \partial_{\nu}^{z} \triangle(x-z) .
$$

Using this notation we can rewrite (43) in the form 


$$
\begin{aligned}
& G^{(1)}\left(x_{1}, x_{2} ; x_{3}, x_{4}\right)=i \lambda e^{i \theta}\left(\chi_{0}\left(x_{1}\right) \chi_{0}^{+}\left(x_{3}\right) \otimes \mathcal{S}_{0}\left(x_{2}-x_{4}\right)\right)\left(\exp \left[\int d^{2} z \mathcal{K}^{\mu}\left(z ; x_{1}, x_{3}\right) \frac{\delta}{\delta J^{\mu}(z)}\right]\right. \\
& \left.\otimes \exp \left[i \int d^{2} z \mathcal{J}_{\mu}\left(z ; x_{2}, x_{4}\right) \varepsilon^{\mu \nu} \partial_{\nu} b^{(1)}(z)+\int d^{2} z \mathcal{J}^{\mu}\left(z ; x_{2}, x_{4}\right) \frac{\delta}{\delta J^{\mu}}\right]\right) \times \\
& \exp \left[\frac{i}{2} \int d^{2} x b^{(1)} \square\left(\square+e^{2} / \pi\right) b^{(1)}-\frac{i}{2} \int d^{2} x d^{2} y\left(J^{\mu}(x)+\varepsilon^{\mu \alpha}\left(\square+e^{2} / \pi\right) \partial_{\alpha} b^{(1)}(x)\right) \times\right. \\
& \left.\triangle_{\mu \nu}(x-y)\left(J^{\nu}(y)+\varepsilon^{\nu \beta}\left(\square+e^{2} / \pi\right) \partial_{\beta} b^{(1)}(y)\right)\right]+ \text { antisymmetrization }
\end{aligned}
$$

where the 'antisymmetrization' is defined by substitutions in (43). The obvious matrical notation has been introduced above to simplify the expression and avoid explicitly writing four spinor indices. (One should keep in mind that both currents $\mathcal{K}$ and $\mathcal{J}$ are matrices in spinor space.) The functional derivatives over current $J$, similarly as in the expressions of the previous section, lead to shifts of $J$ and we obtain

$$
\begin{aligned}
& G^{(1)}\left(x_{1}, x_{2} ; x_{3}, x_{4}\right)=i \lambda e^{i \theta}\left[\chi _ { 0 } ( x _ { 1 } ) \chi _ { 0 } ^ { + } ( x _ { 3 } ) \otimes \mathcal { S } _ { 0 } ( x _ { 2 } - x _ { 4 } ) \operatorname { e x p } \left(i \int d^{2} z \mathcal{J}_{\mu}\left(z ; x_{2}, x_{4}\right) \times\right.\right. \\
& \left.\left.\varepsilon^{\mu \nu} \partial_{\nu} b^{(1)}(z)\right)\right] \exp \left[\frac{i}{2} \int d^{2} x b^{(1)} \square\left(\square+e^{2} / \pi\right) b^{(1)}-\frac{i}{2} \int d^{2} x d^{2} y\left(\mathcal{K}^{\mu}\left(x ; x_{1}, x_{3}\right) \otimes \mathbb{1}+\right.\right. \\
& \left.\mathbb{1} \otimes \mathcal{J}\left(x ; x_{2}, x_{4}\right)+\varepsilon^{\mu \alpha}\left(\square+e^{2} / \pi\right) \partial_{\alpha} b^{(1)}(x)\right) \triangle_{\mu \nu}(x-y)\left(\mathcal{K}^{\nu}\left(y ; x_{1}, x_{3}\right) \otimes \mathbb{1}+\right. \\
& \left.\left.\mathbb{1} \otimes \mathcal{J}\left(y ; x_{2}, x_{4}\right)+\varepsilon^{\nu \beta}\left(\square+e^{2} / \pi\right) \partial_{\beta} b^{(1)}(y)\right)\right]+ \text { antisymmetrization },
\end{aligned}
$$

In the last exponent of (47) we recognize the expressions similar to those of (37) although the tensor structure is now much more perplexed. Nevertheless all functions are known and the evaluation of (47) is only a matter of patience. We do not intend to go into details and only indicate the main points.

1. The term quadratic in $\mathcal{K}$ is just that of the previous section multiplied (tensor product) by 11 .

2. The term quadratic in $\mathcal{J}$ was calculated in our previous work [19].

3. The two terms containing squares of $b^{(1)}$ cancel each other.

4. The terms containing products of $\mathcal{J}$ and $b^{(1)}$ cancel with the first exponent in (47).

5. The terms containing products of $\mathcal{K}$ and $b^{(1)}$ cancel with the appropriate part of the coefficient $\chi_{0}(x) \chi_{0}^{+}(y)$ (see Section [IB).

6. The product terms of both currents $\mathcal{K}$ and $\mathcal{J}$ may be evaluated in the straightforward way and we obtain

$$
i e^{2} \gamma^{5} \otimes \gamma^{5}\left[\beta\left(x_{1}-x_{4}\right)-\beta\left(x_{1}-x_{2}\right)-\beta\left(x_{2}-x_{3}\right)+\beta\left(x_{3}-x_{4}\right)\right] .
$$

With all above taken into account we can now the arising exponent in (47) give the following simple form 


$$
\begin{aligned}
\exp \left[a \mathbb{1} \otimes \mathbb{1}+b \gamma^{5} \otimes \gamma^{5}\right]= & \frac{1}{2}\left(\mathbb{1} \otimes \mathbb{1}-\gamma^{5} \otimes \gamma^{5}\right) \exp (a-b)+ \\
& \frac{1}{2}\left(\mathbb{1} \otimes \mathbb{1}+\gamma^{5} \otimes \gamma^{5}\right) \exp (a+b),
\end{aligned}
$$

where $a$ and $b$ are certain functions expressible by combinations of $\beta$ 's.

The calculations for $k=-1$, which are practically identical, allow us to write down the final expression for the one instanton contributions to $G$

$$
\begin{aligned}
& G^{(+1)}\left(x_{1}, x_{2} ; x_{3}, x_{4}\right)+G^{(-1)}\left(x_{1}, x_{2} ; x_{3}, x_{4}\right)=-\frac{i e}{8 \pi^{3 / 2}} e^{\gamma_{E}}\left(\cos \theta-i \gamma^{5} \sin \theta\right) \otimes \mathcal{S}_{0}\left(x_{2}-x_{4}\right) \times \\
& {\left[\left(\mathbb{1} \otimes \mathbb{1}-\gamma^{5} \otimes \gamma^{5}\right) e^{i e^{2}\left[\beta\left(x_{1}-x_{3}\right)-\beta\left(x_{2}-x_{4}\right)-\beta\left(x_{1}-x_{4}\right)+\beta\left(x_{1}-x_{2}\right)+\beta\left(x_{2}-x_{3}\right)-\beta\left(x_{3}-x_{4}\right)\right]}+\right.} \\
& \left.\left(\mathbb{1} \otimes \mathbb{1}+\gamma^{5} \otimes \gamma^{5}\right) e^{i e^{2}\left[\beta\left(x_{1}-x_{3}\right)-\beta\left(x_{2}-x_{4}\right)+\beta\left(x_{1}-x_{4}\right)-\beta\left(x_{1}-x_{2}\right)-\beta\left(x_{2}-x_{3}\right)+\beta\left(x_{3}-x_{4}\right)\right]}\right] \\
& + \text { antisymmetrization . }
\end{aligned}
$$

In the sector $k=2$ we use again the formula (41). There are now two zero modes. This means that after functional integration over Grassmann variables, analogous to that performed to obtain (31), the product of two $\eta$ 's and two $\bar{\eta}$ 's appears. These four sources have to saturate all four derivatives in (41), since otherwise we would get zero after setting $\eta, \bar{\eta}, J=0$. That in turn means that, in the formula for $G^{(2)}$, the current $\mathcal{J}$ will not appear and

$$
\begin{aligned}
& G^{(2)}\left(x_{1}, x_{2} ; x_{3}, x_{4}\right)=-\lambda^{2} e^{2 i \theta} \chi_{0}\left(x_{1}\right) \chi_{0}^{+}\left(x_{3}\right) \otimes \chi_{1}\left(x_{2}\right) \chi_{1}^{+}\left(x_{4}\right) \exp \left[\frac{i}{2} \int d^{2} x b^{(2)} \square\left(\square+e^{2} / \pi\right) b^{(2)}\right. \\
& -\frac{i}{2} \int d^{2} x d^{2} y\left(\mathcal{K}^{\mu}\left(x ; x_{1}, x_{3}\right) \otimes \mathbb{1}+\mathbb{1} \otimes \mathcal{K}\left(x ; x_{2}, x_{4}\right)+\varepsilon^{\mu \alpha}\left(\square+e^{2} / \pi\right) \partial_{\alpha} b^{(2)}(x)\right) \\
& \left.\triangle_{\mu \nu}(x-y)\left(\mathcal{K}^{\nu}\left(y ; x_{1}, x_{3}\right) \otimes \mathbb{1}+\mathbb{1} \otimes \mathcal{K}\left(y ; x_{2}, x_{4}\right)+\varepsilon^{\nu \beta}\left(\square+e^{2} / \pi\right) \partial_{\beta} b^{(2)}(y)\right)\right] \\
& + \text { antisymmetrization. }
\end{aligned}
$$

We are not going to repeat below the steps leading to the final result, since the modification of previous formulae is only slight. The 2-instanton configurations contribute then in the following way

$$
\begin{aligned}
& G^{(+2)}\left(x_{1}, x_{2} ; x_{3}, x_{4}\right)+G^{(-2)}\left(x_{1}, x_{2} ; x_{3}, x_{4}\right)=-\frac{e^{4}}{256 \pi^{4}} e^{4 \gamma_{E}}\left[e^{2 i \theta}\left(\mathbb{1}-\gamma^{5}\right) \otimes\left(\mathbb{1}-\gamma^{5}\right)\left(x_{2}^{0}+x_{2}^{1}\right)\right. \\
& \left.\left(-x_{4}^{0}+x_{4}^{1}\right)+e^{-2 i \theta}\left(\mathbb{1}+\gamma^{5}\right) \otimes\left(\mathbb{1}+\gamma^{5}\right)\left(-x_{2}^{0}+x_{2}^{1}\right)\left(x_{4}^{0}+x_{4}^{1}\right)\right] \exp \left\{i e ^ { 2 } \left[\beta\left(x_{1}-x_{4}\right)+\right.\right. \\
& \left.\left.\beta\left(x_{2}-x_{3}\right)+\beta\left(x_{1}-x_{2}\right)+\beta\left(x_{3}-x_{4}\right)+\beta\left(x_{1}-x_{3}\right)+\beta\left(x_{2}-x_{4}\right)\right]\right\}+ \text { antisymmetrization } .
\end{aligned}
$$

If we now expand the tensor notation of (50) and (52) into explicit spinor indices and perform the full antisymmetrization (which also restores the apparently broken Lorentz invariance) as defined by (43) we can collect together all the contributions

$$
\begin{aligned}
G_{a b, c d}\left(x_{1}, x_{2} ; x_{3}, x_{4}\right)= & G_{a b, c d}^{(0)}\left(x_{1}, x_{2} ; x_{3}, x_{4}\right)+G_{a b, c d}^{(+1)}\left(x_{1}, x_{2} ; x_{3}, x_{4}\right)+G_{a b, c d}^{(-1)}\left(x_{1}, x_{2} ; x_{3}, x_{4}\right)+ \\
& G_{a b, c d}^{(+2)}\left(x_{1}, x_{2} ; x_{3}, x_{4}\right)+G_{a b, c d}^{(-2)}\left(x_{1}, x_{2} ; x_{3}, x_{4}\right) .
\end{aligned}
$$


Since we now dispose the complete formula for the 4-fermion function, we can extend the analysis carried out in [19], regarding the possible existence of the Schwinger pole in the $t$-channel (the quark-antiquark scattering), over contributions from higher topological sectors. To this goal we identify the appropriate co-ordinates of incoming and outgoing particles: $u \stackrel{\text { def }}{=} x_{1}=x_{3}, v \stackrel{\text { def }}{=} x_{2}=x_{4}$ and perform the Fourier transform of the expression in the new variable $z \stackrel{\text { def }}{=} v-u$. This identification (which should be treated as an appropriate limit) has to be done in a symmetrical way as described in the quoted paper. After these manipulations we see that the formula (45) from [19 acquires additional terms from the $k \neq 0$ instanton sectors

$$
\begin{aligned}
G_{\text {polar }}(k)= & -\frac{i}{4 \pi} \gamma^{0} \otimes \gamma^{0} \frac{\left(k^{1}\right)^{2}}{\left(k^{2}-e^{2} / \pi\right)}+\frac{i e}{4 \pi^{3 / 2}} e^{\gamma_{E}}\left(\gamma^{0} \otimes \gamma^{5} e^{-i \theta \gamma^{5}}-\gamma^{5} e^{-i \theta \gamma^{5}} \otimes \gamma^{0}\right) \times \\
& \frac{k^{1}}{\left(k^{2}-e^{2} / \pi\right)}+\frac{i e^{2}}{8 \pi^{2}} e^{2 \gamma_{E}} e^{-i \theta \gamma^{5}} \otimes e^{-i \theta \gamma^{5}} \cdot\left[\mathbb{1} \otimes \mathbb{1}+\gamma^{5} \otimes \gamma^{5}\right] .
\end{aligned}
$$

We would like to point out here certain observation. If one considers the time ordered product of two $\Psi$ 's and two $\bar{\Psi}$ 's then, for some choice of time arguments, one can introduce a complete set of (out) states obtaining for instance

$$
\sum_{n}<0\left|\Psi\left(x_{1}\right) \bar{\Psi}\left(x_{3}\right)\right| n><n\left|\Psi\left(x_{2}\right) \bar{\Psi}\left(x_{4}\right)\right| 0>.
$$

The set $\mid n>$ is here a Fock set of massive Schwinger bosons since these are the only asymptotic states. The 1-boson contribution, which leads to the appearance of a pole at $k^{2}=e^{2} / \pi$ is expressible, thanks to the formulae of the LSZ formalism, through the vertex function. This in turn means, by virtue of the equation (40), that each of the amplitudes above have the contributions from both $k= \pm 1$ sectors. In consequence, the polar part of $G$ should, beside sectors $+1+1$ and $-1-1$, contain also traces of sectors $+1-1$ and $-1+1$. While calculated explicitly this leads to the substitution $\gamma^{5} \otimes \gamma^{5}$ for $\mathbb{1} \otimes \mathbb{1}$ in the last term of (54). Why such contributions appear neither in $G^{(0)}$ nor in $G^{(2)}$ remains for us presently unclear.

\section{CONCLUSIONS}

The expressions for the Green functions obtained in sections [I] and [II show that the Schwinger Model may be explicitly solved also with all topological effects taken into account. As argued in [19], all higher functions (with no more than 4 external fermion 'legs'), thanks to the two Ward identities, which are preserved in the nonzero instanton sectors too, are the combinations of those found above.

From the formulae (39) and (53) together with (42,50,52) we can obtain, by taking the appropriate limits, the values of the condensates and verify the cluster property. We do not treat, in this work, the restoration of the cluster property as a support for the use of the $\theta$-vacuum instead of a topological one since this fact is well known and needs no verification. We rather show that the obtained formulae are selfconsistent and agree (in this special limit) with former results of other authors.

The values of condensates 


$$
\mathcal{V} \stackrel{\text { def }}{=}<\bar{\Psi}(x) \Psi(x)>_{v a c}, \quad \mathcal{V}_{A} \stackrel{\text { def }}{=}<\bar{\Psi}(x) \gamma^{5} \Psi(x)>_{v a c}
$$

may be easily obtained from eq. (39). If we recall that $\beta(0)=0$ we get

$$
\mathcal{V}=\frac{e}{2 \pi^{3 / 2}} e^{\gamma_{E}} \cos \theta, \quad \mathcal{V}_{A}=-\frac{i e}{2 \pi^{3 / 2}} e^{\gamma_{E}} \sin \theta
$$

which agrees with the results of other authors [10,28] and constitutes the 1-instanton contribution. To verify the clusterization property let us now consider the objects defined by

$$
\mathcal{W}(x, y) \stackrel{\text { def }}{=}<\bar{\Psi}(x) \Psi(x) \bar{\Psi}(y) \Psi(y)>_{v a c}, \quad \mathcal{W}_{A}(x, y) \stackrel{\text { def }}{=}<\bar{\Psi}(x) \gamma^{5} \Psi(x) \bar{\Psi}(y) \gamma^{5} \Psi(y)>_{v a c}
$$

The $k= \pm 1$ does not contribute here since the trace of the product of an odd number of gamma matrices is zero and that is what we obtain while exploiting equation (50). From the sector $k=0$ we obtain

$$
\begin{aligned}
& \mathcal{W}^{(0)}(x, y)=\frac{e^{2}}{8 \pi^{3}} e^{2 \gamma_{E}+2 K_{0}\left(\sqrt{-e^{2}(x-y)^{2} / \pi}\right)}, \\
& \mathcal{W}_{A}^{(0)}(x, y)=-\frac{e^{2}}{8 \pi^{3}} e^{2 \gamma_{E}+2 K_{0}\left(\sqrt{-e^{2}(x-y)^{2} / \pi}\right)},
\end{aligned}
$$

and for $k= \pm 2$ the result is

$$
\mathcal{W}^{(+2 ;-2)}(x, y)=\mathcal{W}_{A}^{(+2 ;-2)}(x, y)=\frac{e^{2}}{8 \pi^{3}} e^{2 \gamma_{E}-2 K_{0}\left(\sqrt{-e^{2}(x-y)^{2} / \pi}\right)} \cos 2 \theta
$$

In particular in the limit $(x-y)^{2} \rightarrow-\infty$, where $K_{0} \rightarrow 0$, we have

$$
\mathcal{W}=\frac{e^{2}}{8 \pi^{3}} e^{2 \gamma_{E}}(1+\cos 2 \theta), \quad \mathcal{W}_{A}=\frac{e^{2}}{8 \pi^{3}} e^{2 \gamma_{E}}(-1+\cos 2 \theta)
$$

and thanks to the identity $\cos 2 \theta=2 \cos ^{2} \theta-1=1-2 \sin ^{2} \theta$ the clusterization reappears

$$
\mathcal{W}=\mathcal{V} \cdot \mathcal{V}, \quad \mathcal{W}_{A}=\mathcal{V}_{A} \cdot \mathcal{V}_{A}
$$

We would like to devote now a few words to the $\theta$ dependence of the Green functions we obtained. It is well known [7,9] that in the case of massless fermions, thanks to the chiral invariance of the Lagrangian, the parameter $\theta$ may be gauged away. This happens because of the close connection between the chiral anomaly and the winding number. That it can actually be done is confirmed by our formulae (39, 42, 50, 52). For each product of $\Psi$ and $\bar{\Psi}$ in a Green function there appears a factor $e^{-i \theta \gamma^{5}}$ in the final formulae which might obviously be cancelled by the appropriate chiral gauge transformation performed on the fermion field.

\section{ACKNOWLEDGMENTS}

The author would like to thank to Professors J. Namysłowski and K. Meissner for interesting and inspiring discussions. 


\section{REFERENCES}

[1] J. Schwinger, in Theoretical Physics, Trieste Lectures 1962 (I.A.E.A., Vienna 1963), p. 89; Phys. Rev. 128, 2425(1962).

[2] G. 't Hooft, Phys. Rev. Lett. 37, 8(1976).

[3] M. Atiyah and I. Singer, Ann. Math. 87, 484(1968).

[4] G. 't Hooft, Phys. Rev. D14, 3432(1976).

[5] J. Kiskis, Phys. Rev. D15, 2329(1977).

[6] M.M. Ansourian, Phys. Lett. 70B, 301(1977).

[7] K. Huang, Quarks, Leptons and Gauge Fields, World Scientific 1982.

[8] C.G. Callan, R.F. Dashen and D.J. Gross, Phys. Lett. 63B, 334(1976).

[9] R. Rajaraman, Solitons and Instantons. An Introduction to Solitons and Instantons in Quantum Field Theory, North-Holland (Amsterdam, New York, Oxford 1982).

[10] C. Adam, Z. Phys. C63, 169(1994).

[11] A.V. Smilga, Phys. Rev. D49, 5480(1994).

[12] C. Gattringer, hep-th/9503137.

[13] K. Stam, J. Phys. G9, L229(1983).

[14] G. Thompson and R. Zhang, J. Phys. G13, L93(1987).

[15] F. J. Dyson, Phys. Rev. 75, 1736 (1949).

[16] J. Schwinger, Proc. Natl. Acad. Sci. USA 31, 452 (1951).

[17] R. Delbourgo and G. Thompson, J. Phys G8, 385(1982).

[18] G. Thompson, Phys. Lett. 131B, 385(1983).

[19] T. Radożycki and J.M. Namysłowski, hep-th/9809129.

[20] J. Spanier and K.B. Oldham, An atlas of functions (HPC-Springer, Washington, Berlin 1987).

[21] K. Fujikawa, Phys. Rev. Lett. 42, 1195(1979); Phys. Rev. D21, 2848(1980).

[22] R. Roskies and F. Schaposnik, Phys. Rev. D23, 558(1981).

[23] C. Adam, R.A. Bertlmann and P. Hofer, Riv. N. Cim 16, 1(1993).

[24] The source term should be written in that way that the functional derivative $\frac{\delta}{i \delta J}$ act only on $e^{i \int d^{2} x J^{\mu} A_{\mu}^{(0)}}$.

[25] G. Maiella and F. Schaposnik, Nucl. Phys. B132, 357(1978).

[26] C. Jayewardena, Helv. Phys. Acta 61, 636(1988).

[27] F.J. Yndurain, Quantum Chromodynamics, (Springer-Verlag, New York, Berlin, Heidelberg, Tokyo 1983).

[28] G. McCartor, Int. J. Mod. Phys. A12, 1091(1997). 